\title{
Foreword to the Special Issue on Ground Penetrating Radar: Modeling Tools, Imaging Methods and Systems Concepts
}

\begin{abstract}
This special issue spreads from the XIII International Conference on Ground Penetrating Radar (GPR2010) held in June 2010 in Lecce, Italy. Ground penetrating radar (GPR) is one of the mostly assessed and exploited technologies for non-destructive subsurface sensing and imaging. As a matter of fact, its flexibility (as far as systems, measurement protocols and processing strategies are concerned) makes it a powerful tool to handle many different observation and sensing applications, ranging from structural monitoring to demining, security and geophysical surveys. Due to the challenging needs arisen by such a widespread application, GPR is an extremely active field for scientific research as indeed efforts aimed at developing new modeling tools, imaging methods and systems concepts can either improve the outcome of GPR surveys or open the way to new, not yet explored, applications. In this respect, this special issue presents a selection of papers that provides a comprehensive and up-to-date overview of the state of the art of research activities carried out on the most relevant and promising trends of development of GPR technology.
\end{abstract}

\section{INTRODUCTION}

$\mathbf{G}$ ROUND penetrating radars (GPRs) have been utilized for non-destructive subsurface sensing and imaging for decades and have wide and well-known application in many areas, such as, for instance, archaeology, geology, glaciology, sedimentology, hydrology, mining/tunneling, and concrete/pavement evaluation. GPR is now a well-assessed technology in surveying and characterizing subsurface geological features such as ice thickness, underground layers, rock beds, fault lines, and lake sediments. More recently, this technique has also gained an increasing importance in planetary exploration missions, as pointed out by the satellite-based platforms already working in Mars exploration and under development for lunar exploration.

GPR offers the highest resolution in subsurface imaging of any method for geophysical applications, approaching centimeters under favourable conditions. Depths of investigation vary from less than a meter to over 3 kilometers depending upon the material properties and operational frequency range. GPRs have been used in airborne surveys, on or near the ground surface, and also in boreholes. There are several companies making commercial equipment around the world, thousands of companies offering GPR services, and many institutions performing research in the field. GPR clearly is an important technology for geoscience and remote sensing applications.

The research area related to GPR is highly active, as witnessed by various special issues of prestigious peer-reviewed international journals published through the years. In particular, the IEEE GRSS Society has covered this topic through a special issue published in the GRS Transactions [1], and this

Digital Object Identifier 10.1109/JSTARS.2011.2177397 special issue, which is the first one published in JSTARS in this framework, reports some results presented at the most recent, very successful, International Conference on Ground Penetrating Radar.

The XIII International Conference on Ground Penetrating Radar (GPR2010) was held in Lecce, Italy, June 21-25, 2010, in the historical and fascinating frame of the castle Carlo V. The Conference, chaired by Dr. Raffaele Persico, had about 250 attendants, among which were many young researchers and about 40 pupils of the School of Specialization in Archaeology of the University of Salento. Fifteen exhibitions and several sponsorships, further than several patronages, among which those particularly prestigious from UNESCO, Red Cross, and WWF, witnessed the interest of the GPR technique for cultural heritage, humanitarian and environmental applications. Within the conference, three tutorials and a workshop were organized and four invited talks were held, focused on the following: the history and the future of the GPR; the planetary exploration with a focus on Mars observation; glaciology issues; and inversion methodologies for GPR data processing. Moreover, two young researchers, Dr. Anja Klotzsche and Dr. Kazunori Takahashi, were awarded by an international review committee, chaired by Dr. Francesco Soldovieri, for the quality of the work they presented at the conference.

For the technical program of GPR2010, nearly 180 papers were presented in both the oral (the largest part) and the interactive sessions. The review process was very accurate thanks to the work of about 90 reviewers, coordinated by three Technical Co-Chairs, Dr. Lorenzo Crocco, Prof. Luciana Orlando, and Prof. Massimiliano Pieraccini. The Proceedings are now available through the IEEE digital repository [2].

\section{New Modeling Tools, Imaging Methods and Systems CONCEPTS FOR GROUND PENETRATING RADAR}

Taking into account the quality of the papers presented at the GPR2010 conference, and also relying on the response to an open call to the scientific community working on GPR, the Guest Editors of this special issue have "distilled" the ones provided in the following pages, which in our opinion provide a good overview of the most relevant and promising trends of development of this technology.

In this respect, the growing number of new and challenging applications of GPR (e.g., mapping of underground infrastructure in urban areas, archaeology, high precision agriculture, antipersonnel landmine detection, through-obstacle imaging and concealed object detection for security purposes, underground imaging for tunnel detection and security borders) have driven improvements in GPR technology and methodologies. These 
applications demand for advancements concerned with modeling tools, needed to deploy an accurate frame for added value model based inversion approaches; imaging methods, needed to provide high resolution and reliable images of the subsurface; hardware systems capable of effectively gathering high diversity data (multi-polarization, multi-fold, etc.) in a stable way (i.e., as less dependent on the scenario as possible).

This special issue contains nine papers that are a significant example of the research and technological efforts to comply with the above-mentioned needs.

The paper by Takahashi et al. [3], related to the one awarded at the conference, tackles a problem particularly important for GPR, that is, the effect of clutter and its modeling. As a matter of fact, unlike traditional radar, the definition of clutter in the framework of GPR is quite subtle due to the intrinsic inhomogeneous nature of the imaged scenario. Thus, the effort of the authors has been that of studying the influence of soil heterogeneity on the detection performance of GPR. Notably, the study has been carried out by applying analytical modeling tools to appraise the outcomes of a controlled experiment.

The paper by Saintenoy and Hopmans [4] shows the importance of suitable modeling for successful GPR data processing. In particular, with respect to the application of GPR to determine the ground water table position, it presents a series of numerical experiments aimed at investigating the relation between GPR measurements and the soil retention's curve. Then, it proposes a simplified piecewise linear model to extract this parameter which is tested through a controlled laboratory experiment.

The paper by Brancaccio et al. [5] moves in the framework of the inverse scattering methods for GPR imaging and is concerned with the theoretical and numerical/experimental analysis of an approach for localizing cylinders under a reflection mode and bistatic configuration. The theoretical expectations are validated by an experimental analysis regarding both perfectly conducting and dielectric cylinders located in free space, where the data are collected by means of an advanced stepped frequency GPR system.

The paper by Ivashov et al. [6] reports on the implementation and exploitation of the holographic based radar technology, a relatively novel technology for the applications of the subsurface and hidden objects imaging. Holographic GPR offers the advantage of a fast diagnostics procedure for imaging of shallower targets and examples of application are shown for mature (civil engineering) and new application fields as security and land mine detection.

The paper by Nakano and Hirose [7] is an interesting contribution in the framework of array antennas for near field imaging. The efforts of the authors are focused on the topic of the mitigation of the coupling among the array elements, which can affect the overall diagnostic procedure. The authors propose a hardware solution as a taper-walled linearly tapered slot antenna for ground penetrating radar system to be applied in plastic landmine detection and characterization.

The paper by Rial et al. [8] is concerned with the GPR systems and antennas measurement and characterization and represents an effort to set-up a strategy to verify the stability of GPR systems performances in terms of the electromagnetic radiated fields. This activity is relevant as a starting point to develop a methodology for calibrating GPR devices and to verify proper operation.

The paper by Liu et al. [9] is concerned with the application of time domain GPR system in an unconventional field as the human being vital signatures detection and characterization, where the focus is to the set-up and analysis in realistic cases of data processing tools able to model the electromagnetic scattering, to mitigate the "static" clutter and perform an analysis of the vital signs by a novel signal processing approach.

The paper by Feng et al. [10] is a relevant contribution to the application of handheld GPR systems in landmine detection. In particular, advanced signal and modelling tools are exploited to tackle the challenging problem of the non-regularspaced measurement scans so to achieve high quality images of the subsurface. The imaging procedures are tested by means of the state-of-the-art dual sensor, advanced landmine imaging system (ALIS).

Finally, the paper by Pallavi et al. [11] describes a case study concerned with the application of GPR to characterize soil moisture content. As is well known, such a parameter is a key variable for environmental and agricultural studies and GPR, which allows to fill the gap between point measurements and remote sensing monitoring, has been indeed widely used in the last years to this end. The interest of this peculiar study resides in the fact that GPR is used for the first time to characterize Japanese Andisol, a peculiar loamy soil originated from volcanic ash.

\section{CONCLUSIONS}

This issue, which is the first one published in JSTARS focused on the topic of Ground Penetrating Radar, continues the tradition of Special Issues edited by the IEEE GRSS Society devoted to research in this field. The papers that compose this special issue show the vitality and the multidisciplinary nature of the research activities on this topic. Hence, we hope the forthcoming GPR2012 conference will be covered as well by another contribution to scientific research in this field.

\section{ACKNOWLEDGMENT}

The Guest Editors would like to thank all the referees that have been involved in the thorough review process that has lead to selection of the papers included in this issue. In particular, we would like to thank Dr. Raffaele Persico and Dr. Kun Shan Chen, for their valuable help in managing the review process of the submitted papers. 
LORENZO CROCCO, Guest Editor

CNR - National Research Council of Italy

IREA-Institute for Electromagnetic

Sensing of the Environment

Naples, 80124 Italy

crocco.1@irea.cnr.it

Massimiliano Pieraccini, Guest Editor

Department of Electronics and

Telecommunications

University of Florence

Florence, 50139 Italy

massimiliano.pieraccini@unifi.it

\section{Motoyuki SATO, Guest Editor}

Center for Northeast Asian Studies

Tohoku University

Sendai, 980-8576 Japan

sato@cneas.tohoku.ac.jp

FRANCESCO SOLDOVIERI, Guest Editor

CNR - National Research Council of Italy

IREA - Institute for Electromagnetic

Sensing of the Environment

Naples, 80124 Italy

soldovieri.f@irea.cnr.it

\section{REFERENCES}

[1] C.-C. Chen, J. T. Johnson, M. Sato, and A. G. Yarovoy, "Foreword to the special issue on subsurface sensing using ground-penetrating radar (GPR)," IEEE Trans. Geosci. Remote Sens., vol. 45, no. 8, pp. 2419-2421, Aug. 2007.

[2] Proc. XIII Int. Conf. Ground Penetrating Radar, GPR2010, L. Crocco, L. Orlando, and M. Pieraccini, Eds. [Online.] Available: http://ieeex-

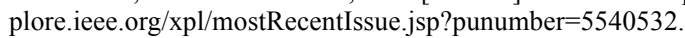

[3] K. Takahashi, J. Igel, and H. Preetz, "Clutter modeling for groundpenetrating radar measurements in heterogeneous soils," IEEE J. Sel. Topics Appl. Earth Observ. Remote Sens., vol. 4, no. 4, Dec. 2011.

[4] A. Saintenoy and J. W. Hopmans, "Ground penetrating radar: Water table detection sensitivity to soil water retention properties," IEEE J. Sel. Topics Appl. Earth Observ. Remote Sens., vol. 4, no. 4, Dec. 2011.

[5] A. Brancaccio, C. D. Dio, and G. Leone, "Assessment of an inverse algorithm for cylinders localization," IEEE J. Sel. Topics Appl. Earth Observ. Remote Sens., vol. 4, no. 4, Dec. 2011.

[6] S. I. Ivashov, V. V. Razevig, I. A. Vasiliev, A. V. Zhuravlev, T. D. Bechtel, and L. Capineri, "Holographic subsurface radar of RASCAN type: Development and applications," IEEE J. Sel. Topics Appl. Earth Observ. Remote Sens., vol. 4, no. 4, Dec. 2011.

[7] Y. Nakano and A. Hirose, "Taper-walled linearly tapered slot antenna," IEEE J. Sel. Topics Appl. Earth Observ. Remote Sens., vol. 4, no. 4, Dec. 2011.

[8] F. I. Rial, H. Lorenzo, A. Novo, and M. Pereira, "Checking the signal stability in GPR systems and antennas," IEEE J. Sel. Topics Appl. Earth Observ. Remote Sens., vol. 4, no. 4, Dec. 2011.

[9] L. Liu, Z. Liu, and B. E. Barrowes, "Through-wall bio-radiolocation with UWB impulse radar: Observation, simulation and signal extraction," IEEE J. Sel. Topics Appl. Earth Observ. Remote Sens., vol. 4, no. 4, Dec. 2011.

[10] X. Feng, M. Sato, and C. Liu, "Hand-held GPR imaging using migration for irregular data," IEEE J. Sel. Topics Appl. Earth Observ. Remote Sens., vol. 4, no. 4, Dec. 2011.

[11] B. Pallavi, H. Saito, and M. Kato, "On mapping surface moisture content of Japanese Andisol using GPR," IEEE J. Sel. Topics Appl. Earth Observ. Remote Sens., vol. 4, no. 4, Dec. 2011.

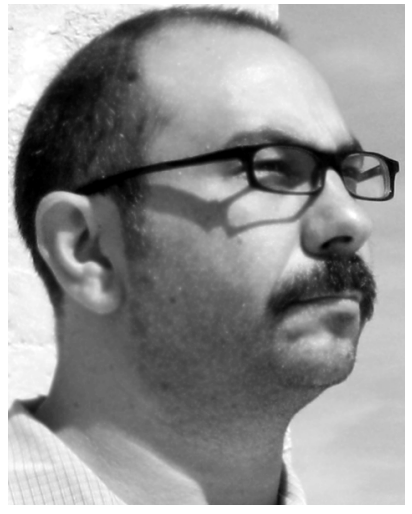

Lorenzo Crocco (M'05-SM'10) was born in Naples, Italy, in 1971. He received the Laurea degree (summa cum laude) in electronic engineering and the Ph.D. degree in applied electromagnetics from the University of Naples "Federico II," Naples, in 1995 and 2000, respectively.

Since 2001, he has been a Research Scientist with the Institute for Electromagnetic Sensing of the Environment, National Research Council (IREA-CNR), Naples. Since 2009, he has also been an Adjunct Professor at the Mediterranea University of Reggio Calabria, Italy. His scientific interests include forward and inverse electromagnetic scattering problems, quantitative and qualitative imaging methods, as applied to noninvasive diagnostics, ground-penetrating radar, microwave breast cancer imaging, and photonic bandgap devices. On these topics, Dr. Crocco has lead or participated to several national and international research projects and has authored more than 50 scientific publications in peer-reviewed journals and more than 200 conference publications. He has edited the proceedings book of the IV International Workshop on Advanced Ground Penetrating Radar (IWAGPR2007) and of the XIII International Conference on ground Penetrating Radar (GPR2010), both under IEEE sponsorship. He has also been guest editor of a two special issues of Near Surface Geophysics $(2008,2010)$ dedicated to GPR. He has also been guest editor of a special section of Inverse Problems (2009) dedicated experimental validation of microwave imaging methods.

Dr. Crocco has co-chaired the IV International Workshop on Advanced Ground Penetrating Radar (Naples, Italy, 2007), and has been Technical Co-Chair of the XIII International Conference on ground Penetrating Radar (Lecce, Italy, 2010). He is a Fellow of The Electromagnetics Academy. He was the recipient of the G. Barzilai Award for Young Scientists from the Italian Electromagnetic Society in 2004 and the Young Scientist Award at the XXVIII International Union of Radio Science General Assembly in 2005. In 2009, he was awarded as one of the top one hundred under-40 scientists of CNR. 


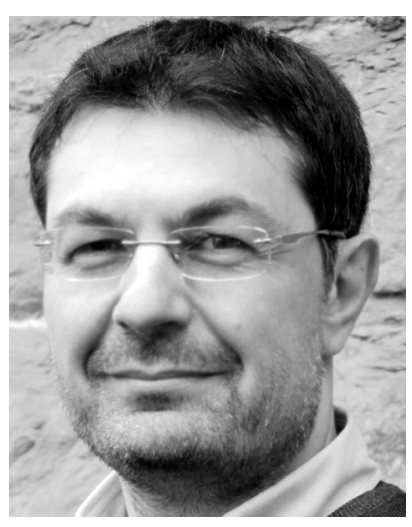

Massimiliano Pieraccini graduated in physics in 1994 ("Nello Carrara" degree prize) at the University of Florence, Italy, and received the Ph.D. degree in non-destructive testing in 1998.

In 1995, he joined the Department of Electronics and Telecommunications of the University of Florence, where in 1997 he gained the permanent position of Assistant Professor. Since 2005, he has been an Associate Professor. He has been principal investigator and manager of several research projects funded by European Community, Italian Research Ministry, and private companies. He manages a research group of five people. He is author of about 100 scientific articles, including more than 50 articles on peer-reviewed international journals. He has taught Basic Electronics, Electronics of Telecommunications, and Technology for Cultural Heritage at the University of Florence. His research interests has included optoelectronic sensors, ultrasound transducers, 3D acquisition systems, interferometric radar, and ground penetrating radar.

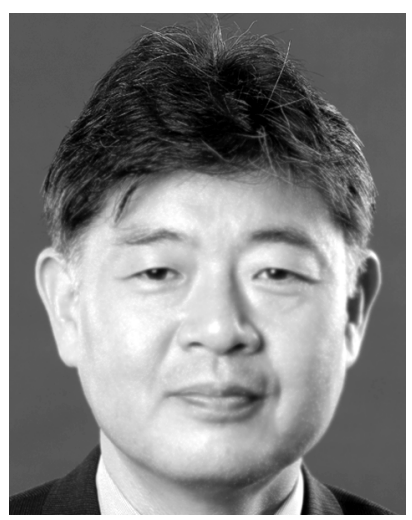

Motoyuki Sato (S'79-M'80-SM'02-F'10) received the B.E., M.E., and Dr. Eng. degrees in information engineering from Tohoku University, Sendai, Japan, in 1980, 1982, and 1985, respectively.

Since 1997, he has been a Professor at Tohoku University, and a Distinguished Professor of Tohoku University since 2007. He has been the Director of the Center for Northeast Asian Studies, Tohoku University, since 2009. From 1988 to 1989, he was a visiting researcher at the Federal German Institute for Geoscience and Natural Resources (BGR) in Hannover, Germany. His current interests include transient electromagnetics and antennas, radar polarimetry, ground penetrating radar (GPR), borehole radar, electromagnetic induction sensing, and interferometric and polarimetric SAR. He has conducted the development of GPR sensors for humanitarian demining, and his sensor ALIS, which is a handheld dual sensor, has detected more than 80 mines in mine fields in Cambodia since May 2009. He has been a visiting Professor at Jilin University, China, Delft University of Technology, The Netherlands, and the Mongolian University of Science and Technology.

Dr. Sato has been a member of the GRSS AdCom since 2006, where he is responsible for specialty symposia and Asian issues. $\mathrm{He}$ is an associate editor of IEEE GRSL, and was a guest editor of the special issue of GPR2006 and GPR2010 in the IEEE Transactions On GeOSCIENCE AND REMOte SENSING. He was the chair of the IEEE GRSS Japan Chapter in 2006-2007. He is the general chair of IGARSS 2011.

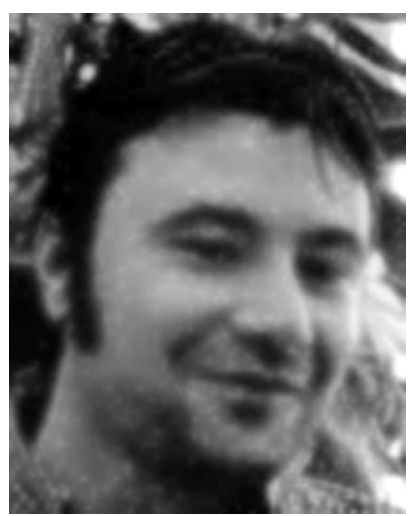

Francesco Soldovieri received the Laurea degree in electronic engineering from the University of Salerno in 1992 and the Ph.D. degree in electronic engineering in 1996 from University of Naples "Federico II", Naples, Italy. In 1993, he joined the Electromagnetic Research Group of University of Naples "Federico II" first as a Ph.D. student and then on a Postdoctoral Fellowship (1998/1999).

In 1999-2000, he collaborated with the Research Group on Applied Electromagnetics of the Second University of Naples by performing activities in antennas diagnostics and inverse scattering problems. From 2001 to 2006, he was a Research Scientist at the Institute for Electromagnetic Sensing of the Environment of the Italian National Research Council (IREA-CNR). Since 2006, being the winner of a national competition, he is a Senior Research Scientist at IREA. His main scientific interests include GPR applications, electromagnetic diagnostics, inverse scattering, antenna diagnostics and characterization. He is a coauthor of about 130 papers in national and international journals and more than 140 conference papers.

Dr. Soldovieri has been a member of scientific committees and technical review panels for the International Conference on Ground Penetrating Radar in 2004, 2006, and 2008, and for the International Workshop on Advanced Ground Penetrating Radar in 2003, 2005, and 2009. He has served as reviewer for IGARSS in 2008 and 2009. He was General Chair of the International Workshop on Advanced Ground Penetrating Radar 2007 held in Naples, Italy. He was appointed as General Co-Chair of the International Conference on Ground Penetrating Radar held in Lecce, Italy in 2010. He has been a convener of Special Sessions at PIERS (2005) and the EGU General Assembly (2007, 2008, 2009). Dr. Soldovieri edited the proceedings book of the IV International Workshop on Advanced Ground Penetrating Radar (IWAGPR2007) under IEEE sponsorship. He has been Guest Editor for Special Issues on Advances in Geosciences (Nov. 2008), Near Surface Geophysics (Dec. 2008), Journal of Applied Geophysics (March 2009), and Journal of Geophysics and Engineering (2010, 2011) on topics concerned with GPR. He is a member of the editorial board for IEEE Geoscience and Remote Sensing Letters and Journal of Geophysics and Engineering. Dr. Soldovieri was awarded with the 1999 Honorable Mention for the H.A. Wheeler Applications Prize Paper Award of the IEEE Antennas and Propagation Society. 